\title{
Efficiency of Multislice Computed Tomography in Diagnosis of Coronary Artery Disease compared to Cardiac Catheterization
}

\author{
Nagwan Elhussein ${ }^{1}$, Rania Mohammed Ahmed ${ }^{2}$, Rawan AL-Thobity ${ }^{2}$, Hadeel Al-Qurashi ${ }^{2}$, Shahad AL-Sulimani ${ }^{2}$, \\ Shmookh AL-Turgi ${ }^{2}$, Wasyf AL-Zaidi ${ }^{2}$, Basma Al-Sufyani ${ }^{2}$, Mameedullah Kazim ${ }^{3}$ \\ ${ }^{1}$ Radiological Science Department,- Alghad international college for Applied Medical Science, Riyadh. Saudi Arabia \\ ${ }^{2}$ Radiological Science Department, College of Applied Medical Science - Taif University, Saudi Arabia \\ ${ }^{3}$ Cardiology Department, Alhada Military hospital, Taif City, Saudi Arabia
}

*Corresponding author: Dr. Nagwan Elhussein,nagwanelhussein@ hotmail.com

Received 31 January 2019;

Accepted 19 February 2019;

Published 23 February 2019

\begin{abstract}
Coronary artery disease (CAD) is a leading cause of mortality and morbidity in developed countries, although percutaneous coronary intervention and coronary artery bypass grafting have developed recently, also multislice computed tomography has been accepted tool for diagnosing of (CAD). Objective: To assess the efficiency of multislice computed tomography in diagnosing coronary heart disease compared with cardiac catheterization. $\underline{\text { Method: }}$ This retrospective study was conducted at Alhada Military hospital in Taif City, Saudi Arabia, in radiology and cardiac catheterization departments during period from January to April 2018. The study was done by collecting of 51 patients reports of computed tomography and coronary artery catheterization most of them complain of chest pain. Data was analyzed using statistical package for social sciences program (SPSS) version 21.00. And achieved in tables and graphs in percentages according to the age, gender, patient history, symptoms, CT finding and catheterization finding, from the check list. $\underline{\text { Results: }}$ The most common affected age group was (61$80) \mathrm{y}$, male were (52.9\%), the most complain was chest pain. CT scan positive findings were found to be in $(72.5 \%)$ of the patients, while the cardiac catheterization diagnosed (CAD) in (68.6\%) of the patients, there are statistical significance difference between the CT and cardiac catheterization finding $(\mathrm{P}=0.00)$, but there were no statistical significance difference between cardiac catheterization finding with age and gender among study sample. Conclusion: CT scan can accurately diagnose the coronary artery disease, which is an ideal non-invasive technique compared with cardiac catheterization.
\end{abstract}

Keywords: Coronary artery disease, Multislice CT scan, Cardiac catheterization.

\section{Introduction}

Coronary artery disease is defined as acute or chronic form of cardiac disability arising from imbalance between the myocardial supply and demand for oxygenated blood since narrowing or obstruction of the coronary arterial system is the most common cause of myocardial anoxia. ${ }^{[1]}$ Arteries, the most prevalent being atherosclerosis accounting for more than $90 \%$ cases, while other causes are responsible for less than $10 \%$ cases of CAD. Therefore, it is convenient to consider the etiology of CAD under three broad headings: coronary atherosclerosis, superadded changes in coronary atherosclerosis and non-atherosclerotic causes. ${ }^{[1]}$ The speed and degree of luminal narrowing determine whether an atherosclerotic lesion causes significant and clinically evident ischemia. Temporary oxygen insufficiency causes angina pectoris feeling of severe chest pain that may radiate to the neck, jaw, and left arm (some time both arms) and that is often associated with the sensation of chest tightness or suffocation. ${ }^{[2]}$ Occlusion of coronary artery deprives an area of myocardial of its blood supply and leads to the death of muscle cell (myocardial infarction) in the area of vascular distribution. The size of coronary artery that is occluded and the myocardium that it supplies determines the extent of heart muscle damage. ${ }^{[2]}$ Coronary artery diseases are result of dyslipidemias, hypertension, diabetes mellitus and smoking, increasing age, male sex, genetic abnormalities, and familial and racial predisposition. ${ }^{[1]}$ One study reported by (Simon Barquera,et al,2015) They reported that ; the absolute number of deaths due to athermanous coronary disease ACD increased substantially since 1990. However, the age-standardized cardiovascular mortality rates per 100,000 inhabitants decreased globally. High-income countries 
were able to reduce their CVD mortality by $42 \%$ during this time period, whereas two regions-Latin America and Caribbean, and North Africa and Middle East-reduced this outcome by 24 and 23\%, respectively. ${ }^{[3]}$ Finally, three regions: sub-Saharan Africa, Southeast Asia, East Asia and Oceania, and Central Europe, Eastern Europe and Central Asia decreased their mortality rates by $13 \%$ or less in the same period and some of the countries within these regions showed no reductions in this indicator during the last 20 years. Central Europe, Eastern Europe and Central Asia were the world regions with the highest current age-standardized CVD mortality rates, which are more than twice those of Latin America and the Caribbean. ${ }^{[3]}$ Some countries have shown very promising results, for example, the Czech Republic reduced its mortality by $45 \%$ in the study period although its 2010 CVD mortality rate (256 deaths/100,000 inhabitants) is still above the world average for the same year (234 deaths/100,000 inhabitants). Russia, on the other hand, showed no improvement in the mortality rate and an important increase in the number of DALYs per 100,000 inhabitants during the period ( $9540 / 100,000$ inhabitants), which is almost four times that of the UK (2406 years/100,000 inhabitants). ${ }^{[3]}$ All country regions have decreased their ischemic stroke mortality rates and DALYs/100,000 inhabitants from 1990e2010. From 1990e2010, the trends in mortality rates show important differences by Gross Domestic Product per capita GDPC. For ischemic stroke, mortality rates showed little difference in 1990; however, a reduction was observed during 2010 for highincome countries and minor changes in the lower-income ones, with higher mortality rates. For IHD, during 1990, there was a positive association between mortality and GDPC per capita. ${ }^{[3]}$ Higher-income countries had higher rates compared to those countries with lower income. In 2010, a significant reduction in mortality was observed in high-income countries with almost no change over time for the low-income countries. ${ }^{[3]}$ In a study reported by (Mansour M., et al., 2004), they said The overall prevalence of coronary artery disease CAD in Kingdom of Saudi Arabia (KSA) is $5.5 \%$. A national prevention program at community level as well as high risk groups should be implemented sooner to prevent the expected epidemic of CAD that we are seeing, beginning. Measures are needed to change lifestyle and to address the management of the metabolic syndrome, to reduce modifiable risk factors for CAD. A longitudinal study is needed to demonstrate the importance of reducing modifiable risk factors for $\mathrm{CAD}$ in $\mathrm{KSA}^{[4]} \mathrm{A}$ cardiac $\mathrm{CT}$ scan is a painless imaging test that uses $\mathrm{x}$ rays to take many detailed pictures of your heart and its blood vessels. Computers can combine these pictures to create a three-dimensional (3D) model of the whole heart. ${ }^{[5]}$ This imaging test can help doctors detect or evaluate coronary heart disease, calcium buildup in the coronary arteries, problems with the aorta, problems with heart function and valves, and pericardial disease. ${ }^{[5]}$ The scan usually takes about 15 minutes to complete, but can take more than an hour including preparation time and, if needed, the time to take medicines such as beta blockers to slow your heart rate. Before the test, a contrast dye, often iodine, may be injected into a vein in your arm. This contrast dye highlights your blood vessels and creates clearer pictures. ${ }^{[5]}$ CT coronary angiography (CTCA) is an easily applicable and powerful imaging technique, and the precise evaluation for an ACD is the most important role of (CTCA). ${ }^{[6]}$ The sophisticated evaluation of coronary atherosclerotic disease with (CTCA) provides useful information for proper management of patients as well as simple diagnosis. $^{[6]}$

\section{Material and method}

Research Design: This retrospective study was performed in the period from January to April 2018 in the study setting. In addition cross section study was done through interview of physician during patient's preparation for CAD.

\section{Setting and sample}

The study was done in the radiology and cardiac catheterization departments at Alhada Military hospital in Taif City, Saudi Arabia. Fifty one patient's medical records were collected, most of them complain of chest pain. All the patients were examined by multislice computed tomography and cardiac catheterization together.

\section{Method of data collection}

An observation checklist, for data collection from medical records which was included: age, gender, patient history, symptoms, CT finding and catheterization finding after hospital approval.

\section{Protocol}

\section{CT scan protocol}

Siemens MDCT Scanner (128 slices) figure (3-1) was used in coronary angiography and performed using the ECG-gated acquisition to improve temporal resolution and minimize imaging artifacts caused by cardiac motion during a single breath hold. The patient lays down in supine position on the table with his head first and injection of non-ionic contrast material (Omnipaque 350) through the right anti-cubital vein using 18G I.V Cannula at a flow rate $(6 \mathrm{ml} / \mathrm{sec})$ followed by rapid acquisition of consecutive ultrathin sections $(3 \mathrm{~mm})$ through the thorax to evaluate the coronary arteries and the coronary artery bypass grafts. All coronary arteries were studied at $75 \%$ and $40 \%$ of the cardiac cycle with selective reconstruction of the improperly segments at different phases of the cardiac cycle. The acquired volumetric data was used to reconstruct multi-planar reformatted (MPR) maximal intensity projections (MIP) and volume rendering (VR) images.

\section{Cardiac catheterization}

The procedure is performed in a specifically designed room, very much resembling the operating room, called the catheterization laboratory. Preformed under local anesthesia. The procedure is sterile, and all potential access sites must be disinfected, shaved, and sterilized. The patient lays down in supine position on the cardio-angiograph table, the patients are given 10 $\mathrm{mg}$ diazepam and $25 \mathrm{mg}$ diphenhydramine 30-60 min before the procedure. During the procedure, the patient must be monitored by several systems, and the ECG is absolutely essential. The procedure of arterial puncture is performed using Seldinger technique. Under Philip Bi-blam fluoroscopic guidance, the catheter is advanced into the coronary ostium, and several recordings of the left coronary artery are taken. The best view to see the coronary ostia (both left and right) and to perform the ostial coronary cannulation is left anterior oblique (LAO).

\section{Data analysis}

The data was analyzed using statistical package for social sciences program (SPSS) version 21.00 achieved in tables and graphs in percentages according to the age, gender, patient history, symptoms, CT finding and catheterization finding, from the check list. Cross tabulation between cardiac catheterization finding with 
age and sex, and between CT scan finding and cardiac catheterization finding.

The level of significance for this study was set at $(p=0.05)$ to detect any indication of differences found in the data available.

\section{Results}



Figure 1: Demonstrate the percentage of age groups among patient's reports $\mathrm{N}=51$

*This figure shown the most common age group among patient's reports was (41-60) by percentage $24(47.1 \%$ ), while the lowest age group was (81-100) by percentage (3.9\%).

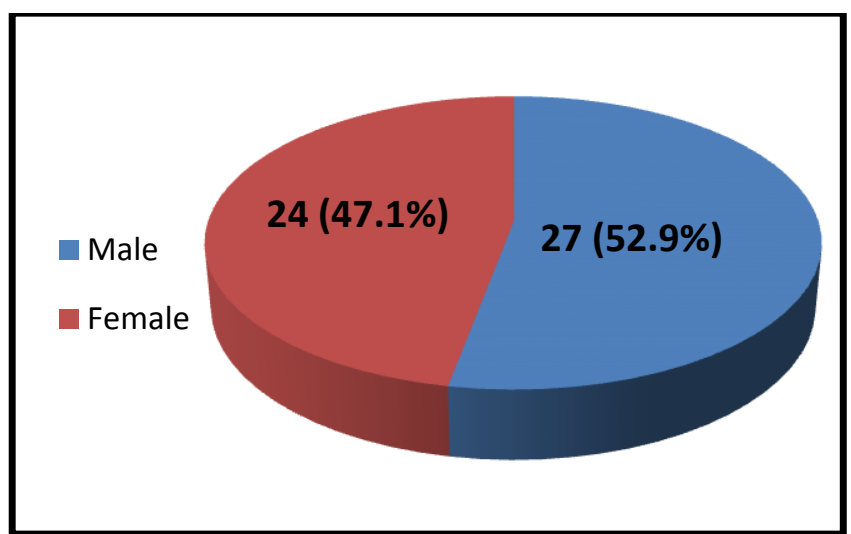

Figure 2: Demonstrate the percentage of gender among patient's reports $\mathrm{N}=\mathbf{5 1}$

* This figure shown the most affected gender was male by percentage $27(52.9 \%$ ) while the female was presented $24(47.1 \%)$.

Table 1: Demonstrate the distribution of patient history among patients reports $\mathrm{N}=\mathbf{5 1}$

\begin{tabular}{|l|l|l|}
\hline Patient history & Frequency & Percent \\
\hline Patient with non-history & 8 & 15.7 \\
Hypertension & 3 & 5.9 \\
Smoker & 2 & 3.9 \\
Diabetes mellitus & 3 & 5.9 \\
Ischemic heart disease & 3 & 5.9 \\
Obesity & 3 & 5.9 \\
Angina & 1 & 2.0 \\
Diabetes mellitus and hypertension & 28 & 54.9 \\
Total & 51 & 100.0 \\
\hline
\end{tabular}

*This table shown the patient history of (Diabetes mellitus and hypertension) represented highest percentage 28(54.9\%), whiles the patient history of angina represented lowest percentage 1 $(2.0 \%)$.

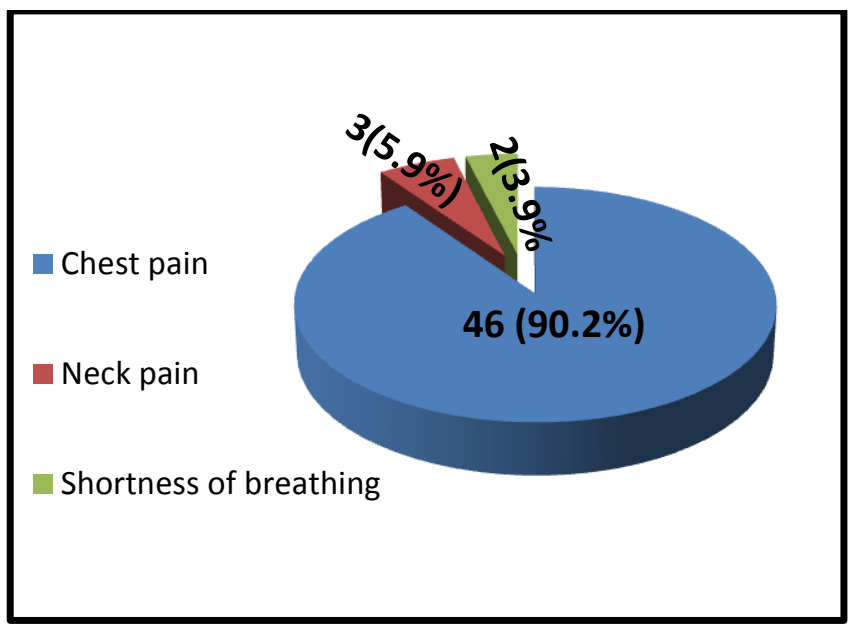

Figure 3: Distribution of patient's symptoms

*This figure shown the chest pain represented higher percentage $46(90.2 \%)$, while the neck pain represented 3(5.9\%) and the shortness of breathing represented $2(3.9 \%)$ as lowest percentage.

Table 2: Shows the CT scan finding N=51

\begin{tabular}{|l|c|c|}
\hline CT finding & Frequency & Percentage \\
\hline Normal & 14 & $27.5 \%$ \\
$\circ \quad$ Normal in Cath. & 10 & $71.4 \%$ \\
$\circ \quad$ CAD in Cath & 4 & $28.6 \%$ \\
\hline Atherosclerosis & 14 & $27.5 \%$ \\
\hline Ischemic heart disease & 8 & $15.7 \%$ \\
\hline Coronary artery stenosis & 14 & $27.5 \%$ \\
\hline Heart failure & 1 & $2.0 \%$ \\
\hline Total of CAD & 37 & $72.5 \%$ \\
\hline Total & 51 & $100.0 \%$ \\
\hline
\end{tabular}

*This table shown the coronary artery diseases was highest percentage $37(72.5 \%)$, and the normal finding represented $14(27.5 \%)$, most of them were also normal in the cardiac catheterization by percentage $10(71.4 \%)$.

Table 3: Demonstrate the cardiac catheterization finding.

\begin{tabular}{|l|c|c|}
\hline Cardiac catheterization finding & Frequency & Percentage \\
\hline Normal & 16 & $31.4 \%$ \\
$\circ \quad$ Normal in CT & 10 & $62.5 \%$ \\
$\circ \quad$ CAD in CT & 6 & $37.5 \%$ \\
\hline \multirow{2}{*}{ Coronary artery disease } & 35 & $68.6 \%$ \\
\cline { 2 - 3 } Atherosclerosis* & 30 & $58.8 \%$ \\
\cline { 2 - 3 } Ischemic heart disease* & 3 & $5.9 \%$ \\
\cline { 2 - 3 } Coronary artery lesion* & 2 & $3.9 \%$ \\
\hline Total & $\mathbf{5 1}$ & $\mathbf{1 0 0 . 0 \%}$ \\
\hline
\end{tabular}

*This table shown the coronary artery diseases was highest percentage $35(68.6 \%)$, and the normal finding represented $16(31.4 \%)$, most of them were also normal in the cardiac catheterization by percentage 10(62.5\%). 
Table 4: Demonstrate the relation between age groups and cardiac catheterization finding

\begin{tabular}{|c|c|c|c|c|c|c|}
\hline \multicolumn{6}{|l|}{ Age } & \multirow{2}{*}{ Total } \\
\hline \multicolumn{3}{|c|}{$20-40$} & $41-60$ & $61-80$ & $81-100$ & \\
\hline \multirow{8}{*}{$\begin{array}{l}\text { Cardiac Catheterization } \\
\text { finding }\end{array}$} & \multirow{2}{*}{$\begin{array}{l}\text { Normal } \\
\text { Total }\end{array}$} & 2 & 12 & 2 & 0 & 16 \\
\hline & & $3.9 \%$ & $23.5 \%$ & $3.9 \%$ & $0.0 \%$ & $31.4 \%$ \\
\hline & \multirow{2}{*}{$\begin{array}{l}\text { Atherosclerosis } \\
\text { Total }\end{array}$} & 3 & 9 & 16 & 2 & 30 \\
\hline & & $5.9 \%$ & $17.6 \%$ & $31.4 \%$ & $3.9 \%$ & $58.8 \%$ \\
\hline & \multirow{2}{*}{$\begin{array}{l}\text { Ischemic heart disease } \\
\text { Total }\end{array}$} & 0 & 2 & 1 & 0 & 3 \\
\hline & & $0.0 \%$ & $3.9 \%$ & $2.0 \%$ & $0.0 \%$ & $5.9 \%$ \\
\hline & \multirow{2}{*}{$\begin{array}{l}\text { Lesion } \\
\text { Total }\end{array}$} & 0 & 1 & 1 & 0 & 2 \\
\hline & & $0.0 \%$ & $2.0 \%$ & $2.0 \%$ & $0.0 \%$ & $3.9 \%$ \\
\hline \multirow{2}{*}{\multicolumn{2}{|c|}{ Total }} & 5 & 24 & 20 & 2 & 51 \\
\hline & & $9.8 \%$ & $47.1 \%$ & $39.2 \%$ & $3.9 \%$ & $100.0 \%$ \\
\hline
\end{tabular}

*This table shown the relation between age groups and cardiac catheterization finding, the age group (61-80) years were most affecting with coronary artery disease with percentage $18(35.04 \%)$ with non-significant relation between them. $N S=0.256$.

Table 5: Demonstrate the correlation between gender and cardiac catheterization finding

\begin{tabular}{|c|c|c|c|c|c|c|c|}
\hline & & & Normal & Atherosclerosis & Ischemic heart disease & Lesion & \\
\hline \multirow[t]{4}{*}{ Gender } & \multirow[t]{2}{*}{ Male } & & 7 & 16 & 2 & 2 & 27 \\
\hline & & Total & $13.7 \%$ & $31.4 \%$ & $3.9 \%$ & $3.9 \%$ & $52.9 \%$ \\
\hline & \multirow{2}{*}{$\begin{array}{l}\text { Femal } \\
\mathrm{e}\end{array}$} & & 9 & 14 & 1 & 0 & 24 \\
\hline & & Total & $17.6 \%$ & $27.5 \%$ & $2.0 \%$ & $0.0 \%$ & $47.1 \%$ \\
\hline \multirow[t]{2}{*}{ Total } & & & 16 & 30 & 3 & 2 & 51 \\
\hline & & & $31.4 \%$ & $58.8 \%$ & $5.9 \%$ & $3.9 \%$ & $100.0 \%$ \\
\hline
\end{tabular}

*This table shown the correlation between gender and cardiac catheterization finding, the male were affected more than female by percentage $20(39.2 \%)$, with non-significant relation between them. $N S=0.466$.

Table 6: Demonstrate the relation between the CT finding and cardiac catheterization finding

\begin{tabular}{|c|c|c|c|c|c|}
\hline & & & \multicolumn{2}{|c|}{ Cath. finding } & \multirow[b]{2}{*}{ Total } \\
\hline & & & normal & CAD & \\
\hline \multirow[t]{4}{*}{ CT finding } & \multirow[t]{2}{*}{ Normal } & & 14 & 0 & 14 \\
\hline & & Total & $27.5 \%$ & $.0 \%$ & $27.5 \%$ \\
\hline & \multirow[t]{2}{*}{ Coronary artery disease } & & 2 & 35 & 37 \\
\hline & & Total & $3.9 \%$ & $68.6 \%$ & $72.5 \%$ \\
\hline \multirow{2}{*}{\multicolumn{2}{|c|}{ Total }} & & 16 & 35 & 51 \\
\hline & & Total & $31.4 \%$ & $68.6 \%$ & $100.0 \%$ \\
\hline
\end{tabular}

*This table demonstrates the relation between the CT finding and cardiac catheterization finding. In 37 cases of 51 reports the CT finding were matched the cardiac catheterization finding by percentage (72.5\%), with significant relation between them. NS=0.000

\section{Discussion}

Coronary artery disease (CAD) is a leading cause of mortality and morbidity in developed countries. ${ }^{[7]} \mathrm{CT}$ imaging are emerging as the most promising complementary imaging techniques in the primary diagnosis of CAD; so the aim of this study to determine the accuracy of CT scan in diagnosing CAD compared with cardiac catheterization by collected of $51 \mathrm{CT}$ and cardiac catheterization reports of patients most of them complain of chest pain in the period from January to April 2018.

The discussion covered the age, gender, history of the patient, symptoms, CT scan finding and cardiac catheterization finding.

Age groups in this study ranged from (24-87) year, age group $(20-40)$ represented $(9.8 \%),(41-60)$ represented $(47.1 \%)$ as highest percentage, $(61-80)$ represented $(39.2 \%)$ and $(81-100)$ represented (3.9\%) as lowest percentage as in figure (1).

Regarding the correlation between the cardiac catheterization finding and the age groups, the researchers found that; The majority of cases diagnosed with $\mathrm{CAD}$ disease were for the age group (61-80) by percentage (35.4\%) while the age group (41-60) were diagnosed with CAD by percentage $(23.5 \%)$ as in table (4) with no significant relation between them ( $\mathrm{NS}=0.256)$, this results was coincidence with (sinqh, et al, 1995) they reported: CAD was significantly higher in the elderly (65 to 84 years) group than in the middle-aged (50 to 64 years) group (168 vs. 97 per 1000), respectively. ${ }^{[8]}$

In this study the majority of cases were male by percentage $(52.9 \%)$ and the female represented(47.1\%) as in figure (2), according to the correlation between gender and the cardiac catheterization finding the researchers found that; the male was affected with CAD more than female by percentage (39.2\%) and $(29.4 \%)$ respectively as in table(5) with no significant relation between them (NS=0.466), this results was not coincidence with (A.H.E.M. Maas,et al, 2010) they said: Cardiovascular disease develops 7 to 10 years later in women than in men and is still the major cause of death in women ${ }^{[9]}$, but it was agreed with (Sophie H., et al, 2017) they reported: While CHD and stroke mortality rates declined considerably between 1980 and 2010 in both sexes, there was some indication for stronger age-specific reductions in CHD in men than women. ${ }^{[10]}$ 
Regarding patient history the researcher found that; patients with more than one diseases like (diabetes mellitus and hypertension) were more likely to get CAD by percentage (54.9\%), (5.9\%) for patient with ischemic heart disease, hypertension, diabetes mellitus and obesity patients, smoker patients represented (3.9\%) percentage and patients with history of angina represented lowest percentage $(2.0 \%)$ as in table(1), this results was not coincidence with (Sultan A.L. Nohair, et al, 2017) they reported: The most common risk factor of CHD among the staff members is obesity by $20.6 \%,{ }^{[11]}$ otherwise our results was agreed with (Mohamed Elsharawy, et al 2008) they showed: There were positive correlations between the prevalence of DM, smoking, dyslipidemia and the severity of presentation of peripheral arterial disease, and the extent of CAD. The correlation between the incidence of DM and the severity of presentation of CAD was also significant. $^{[12]}$

From our results patients with symptoms of chest pain were represented highest percentage $(90.2 \%)$, neck pain represented $(5.9 \%)$ and shortness of breathing represented $(3.9 \%)$ as lowest percentage as in figure (3), this results was agreed with (Sanjeeth Peter,et al 2017) they reported: A majority of the family members were aware about four symptoms of CAD such as tightening of the chest, chest pain, sweating, and shortness of breath. ${ }^{[13]}$

The finding of the CT reports demonstrates 14 cases diagnosed as normal by percentage(27.5\%), also 14 cases diagnosed as atherosclerosis and like them for coronary artery stenosis by percentage $(27.5 \%),(15.7 \%)$ for ischemic heart disease and $(2.0 \%)$ for heart failure as in table (2), in the research written by(Hoffmann U,2016) they said: Noninvasive assessment of coronary artery disease by MDCT has good performance characteristics for ruling out ACS in subjects presenting with possible myocardial ischemia to the emergency department and may be useful for improving early triage. ${ }^{[14]}$

According to the finding of the cardiac catheterization reports the normal patients represented (31.4\%), CAD as general represented $(58.8 \%)$, ischemic heart disease and coronary artery lesion represented $(5.9 \%),(3.6 \%)$ respectively as in table (3).

Finally about the correlation between the CT finding and the cardiac catheterization finding the researchers found that; In 37 cases of $51 \mathrm{CT}$ diagnosis cases matched to cardiac catheterization as in table (5) with highly significant correlation between them (NS=0.00), so the accuracy of the CTA scan to diagnosis CAD was high compared with cardiac catheterization by percentage $(72.5 \%)$ , this result was coincidence with (Amin Arbab et al, 2013) they said: Compared to two other multicenter studies using 64-slice technology, our results revealed slightly lower sensitivity but higher specificity for detecting obstructive CAD by CTA. ${ }^{[15]}$ Also this results was consonant with (Ryotaro Wake, 2013) they reported: Recently, multislice computed tomography has been already accepted as an efficient non-invasive tool for the detection of coronary artery stenosis. ${ }^{[16]}$

\section{Conclusion}

The present study supports that the multislice cardiac CTA scan is very helpful in diagnosing coronary artery disease with very high efficiency, the most common affected age group was (61-80) years and in male rather than female with chest pain symptoms.

\section{Recommendation}

The researcher recommended that; further research should be done with larger sample, and increases the community awareness to prevent and reduce CAD risk factors.

\section{Acknowledgement:}

The authors thank deanship of scientific research, cardiac catheterization and radiology departments, Alhada Military Hospital. Taif City-Saudi Arabia for allowing collection of data to complete this study.

\section{References}

[1] Harsh Mohan, textbook of pathology, 6th ED, (2010), Jaypee Brothers Medical Publishers, India, Chapter1516, pp. 393-429.

[2] Ronald. L. Eisenberg, Nancy. M. Johnson, comprehensive radiographic pathology, 5th Ed, Elsevier, (2012), Ch7, pp. 257- 262.

[3] Simon Barquera,a Andrea Pedroza-Tobias, a Catalina Medina Et al, Global Overview of the Epidemiology of Atherosclerotic Cardiovascular Disease, (July 2015),Archive of medical research, Volume 46, pp. 328338.

[4] Mansour M. Al-Nozha, Mohammed R. Arafah, , Yaqoub , Et al., Coronary artery disease in Saudi Arabia, King Abdul-Aziz University, Jeddah, Kingdom of Saudi Arabia, Saudi Med J, (2004), Vol 25 (9), pp. 1165.

[5] National heart, lung and blood institute, Cardiac CT Scan,2013, Seen on Oct8, (2017) [https://www.nhlbi.nih.gov/health-topics/cardiac-ct-scan]

[6] Tae-Hwan Lim, Practical Textbook of Cardiac CT and MRI, 2015, Springer, Ch5, pp. 63.

[7] R.O. Bonow, L. A. Smaha, S. C. Smith Jr., et al, The international burden of cardiovascular disease, Circulation, (2002), Vol. 106, No. 13, pp. 1602 - 1605.

[8] Sinqh, Niaz MA., Ghosh S., et al, Epidemiological study of coronary artery disease and its risk factors in an elderly urban population of north India, PubMed. journal, (1995) Dec;14(6), pp. 628-34.

[9] A.H.E.M. Maas and Y.E.A. Appelman Gender differences in coronary heart disease, Neth Heart Journal. (2010) Dec; 18(12):, pp. 598-602.

[10] Sophie H Bots, Sanne A E Peters, Mark Woodward, Sex differences in coronary heart disease and stroke mortality, BMJ Glob Health, 2:e000298, pp. 1 -8.

[11] Sultan A.L. Nohair, Abdulrhman A.L. Mohaimeed, Fawzy Sharaf, et al, Risk profile of coronary heart disease among the staff members of Qassim University, Saudi Arabia, IJHS journal, (2017) Jan-Mar;11(1),pp. 15.

[12] Mohamed A Elsharawy and Akram H Alkhadra, Impact of atherosclerosis risk factors on the clinical presentation of arterial occlusive disease in Arabic patients, KSA, Int. Journal Angio, (2008) Winter; 17(4), pp. 203-206.

[13] Sanjeeth Peter, Mahpaekar Mashhadi, Deva J Ajith, et al Awareness about Coronary Artery Disease (CAD) among Relatives of CAD Patients, 2017, 8(8), pp.437441. 
[14] Hoffmann U, Naqurney JT, Moselewski F,et al, Coronary multidetector computed tomography in the assessment of patients with acute chest pain, Circulation, (2006), Vol. 114(25), pp. 2251 - 2260.

[15] Armin Arbab-Zadeh, Julie M. Miller,Carlos E. Rochitte, Diagnostic Accuracy of Computed Tomography Coronary Angiography According to Pre-Test
Probability of Coronary Artery Disease and Severity of Coronary Arterial Calcification, the American College of Cardiology Foundation, Elsevier journal, (2012),59(4).

[16] Ryotaro Wake, Hidetake Iida, Hirohito Ogata,et al, Cardiac computed tomography for the diagnosis of coronary artery atherosclerosis ,International Journal of Clinical Medicine, (2013),Vol. 4, pp,13 - 189. 